\title{
9 Grenzland: Grenze und (lokale) Alltagspraktiken
}

Grenzen sind primär ein räumliches Mittel der Markierung und Abgrenzung politischer, sozialer und ökonomischer Verdichtungen, die die territoriale Form des (modernen) Staats definieren und sichern, ${ }^{1}$ und damit ein wichtiges Element für die Durchsetzung des nationalstaatlichen Raumformats. Dadurch entsteht allerdings immer auch ein neuer Raum: das „Grenzland“, und zwar auf beiden Seiten der Grenze. Seine Bedeutung ist wesentlich. durch die Präsenz der Grenze bestimmt. Diese sei im Grenzland - wie Henk van Houtum, Martine van Kampen und Mark Eker in der Einleitung zu ihrem niederländisch-englischen Atlas- und Essayband „Grensland“ feststellen - zugleich berührbar (,tastbaar“ bzw. „tangible“), wie abstrakt. ${ }^{2}$ Das Grenzland ist also stets (auch) ein Spiegel der Grenzregimes, die es konstituieren.

Grenzregimes werden zumeist begründet mit gesamtstaatlichen („nationalen“) Interessen und Notwendigkeiten oder lassen sich damit in Verbindung bringen: Sie dienen der Kontrolle des Zutritts, der Unterscheidung von legaler und illegaler Migration, dem Schutz des eigenen Finanz-, Waren- und Dienstleistungsmarktes, sollen aber auch z. B. unerwünschte Ausreisen verhindern. Sofern die Kontroll- und Überwachungsfunktionen an die lineare, konkrete räumliche Gestalt der Grenze und ihrer Infrastrukturen gebunden sind, ist die Grenze selbst wie auch das grenznahe Gebiet daher häufig eine Zone besonderer militärischer, polizeilicher und anderer, z. B. fiskalischer Aufmerksamkeit. Das Grenzland ist mithin definiert durch die Funktionen, die eine Staatsgrenze im Hinblick auf die Wahrung staatlicher Souveränität hat. Diese (erwartete, behauptete, angestrebte) Funktionalität korrespondiert auf vielfältige Weise mit der baulichen Gestaltung und planerischen Durchdringung der Grenzlandschaft: Da sind zunächst die Infrastrukturanlagen zur Abschottung und Überwachung (Zäune, Mauern, Kameras, Sendemasten usw.) und Grenzabfertigung selbst zu nennen, die nicht nur beträchtliche Flächen einnehmen können, sondern auch eine enorme symbolische Wirkung im Hinblick auf die Inszenierung des staatlichen Gewaltmonopols

1 J. Painter, „Rethinking Territory“, Antipode 42 (2010) 5, S. 1090 -1118.

2 H. Van Houtum, M. Van Kampen, M. Eker, „Grensland - Het begin / Borderland - The Beginning“ in: M. Eker, H. Van Houtum (Hrsg.), Grensland. Atlas, essays en ontwerp - geschiedenis en toekomst van het grenslandschaft / Borderland. Atlas,Essays and Design - History and Future of the Border Landscape, Wageningen: Uitgeverij Blauwdruk, 2013, S. 14-19, hier S. 16 und 17; H. Donnan, T. M. Wilson, „Territoriality, Anthropology, and the Interstitial: Subversion and Support in European Borderlands“, Focaal - European Journal of Anthropology 41 (2003), S. 9-20. 
haben. Dort, wo Staaten buchstäblich an ihre Grenzen geraten, schlägt sich der Nexus von Territorium, Souveränität und Grenze landschaftlich nieder. Dazu gehören auch Verkehrsflächen, die sowohl der effizienten Lenkung von Güterund Personenströmen wie der schnellen Verlagerung von Grenzschutz, Polizei und Militär dienen, sowie von jeglicher Bebauung und jeglichem Bewuchs freigehaltene Flächen, die klandestine Bewegungen verhindern sollen. Immer wieder haben Fotograf*innen und Journalistinnen die besondere Atmosphäre von Grenzlandschaften einzufangen versucht - nicht zuletzt, weil die in ihnen manifestierte militärisch-polizeiliche Präsenz häufig mit sozialer und ökonomischer Peripherisierung, wenn nicht sogar Devastierung einhergeht. ${ }^{3}$ Die Grenzlandschaft, die wesentlich durch die Sicherung der territorialen Gestalt des Staates geprägt wird, der andere Formen der Nutzung und Gestaltung nach- und untergeordnet sind, produziert daher stets Widersprüche und Kuriosa: Straßen, die im Nichts enden, weil die Verbindung ins Nachbarland fehlt; dysfunktionale Strukturen in der Wasserver- und -entsorgung; unterschiedliche Bestimmungen im Natur- und Landschaftsschutz usw. ${ }^{4}$

Die baulich-planerische Gestaltung des Grenzlandes kann wiederum mit scharfen territorialen Formierungen einhergehen, d. h. das spezifische Interesse des Staates an der Grenze kann sich in rechtlich definierten Abgrenzungen niederschlagen, die mit besonderen Beschränkungen, aber auch Vorrechten verbunden sein können: Dazu gehört die Festlegung von Zonen, die ohne besondere Erlaubnis nicht betreten werden dürfen; die Festlegung von Zollgrenzbezirken, deren Bewohner*innen nur begrenzt Waren aus dem Nachbarland einführen dürfen, oder durch die Festlegung einer Zone, deren Bewohner*innen bestimmte Vorzüge im Hinblick auf den grenzüberschreitenden Personenverkehr genießen,

3 Zum Beispiel zum Niemandsland zwischen Bulgarien und der Türkei: V. Nikolaeva, Simply a Line. No Man's Land Between Bulgaria and Turkey, Rotterdam: NAi Uitgevers, 2009; zur 2004 neuen östlichen Grenze der Europäischen Union: I. Van der Linde, N. Segers, Het einde van Europa. Ontmoetingen langs de nieuwe oostgrens, Rotterdam: Lemniscaat, 2004. Eine Form der Devastierung war auch die physische Abschottung von Orten in der DDR, die in unmittelbarer Nähe der Grenze zur Bundesrepublik lagen.

4 Letzteres hat möglicherweise zunächst Auswirkungen auf das Verhalten von Tieren und nachfolgend dann wirtschaftliche Effekte: Gänse lernen schnell zwischen Vergrämungs- und Ruhegebieten zu unterscheiden und werden auf der Seite der Grenze, auf der die rechtlichen Bestimmungen eine Verfolgung verbieten, ggf. zu einer Belastung für die Landwirtschaft (https:// www.nabu-naturschutzstation.de/de/projekte/naturschutz-ueber-grenzen/wandernde-tiere-ingrenzgebieten [abgerufen am 30 September 2018]). 
weil sie aufgrund ihres residentiellen Status von Visapflichten befreit werden („kleiner Grenzverkehr“). ${ }^{5}$

Im letzten Punkt deutet sich bereits an, dass Grenzländer mehr sind als Räume der Erfüllung staatlicher Sicherungsfunktionen. Sie sind vielmehr Bezugsräume alltäglichen Handelns und daher auch in dessen Logiken eingebunden. Daher wäre es verkürzt, das Grenzland allein auf rechtliche, polizeiliche und administrative Dispositionen zu reduzieren. Alltägliche Praktiken der Aneignung von Grenzen ergeben ein weitaus komplexeres Bild. Arbeiten zum alltäglichen Handeln in Grenzregionen haben betont, dass Grenzen nicht allein und auch nicht zwangsläufig primär als restriktive Institutionen wahrgenommen werden, sondern dass ihre Wahrnehmung in Bezug auf Bedürfnisse, Erwartungen, Ziele und Interessen individuell und gruppenbezogen wie auch zeitlich variiert. Auf welche Weise eine Grenze in Alltagspraktiken relevant gemacht wird, ist dementsprechend breit und kann hier nur grob umrissen werden: Unterschiede im Warenangebot und in der -nachfrage sowie in der Preisbildung, die z. B. durch unterschiedliche Mehrwertsteuersätze begründet sein können, können beispielsweise einerseits Geschäftsgrundlage grenzüberschreitend agierender Unternehmen darstellen, andererseits kann das Nachbarland schlicht als Möglichkeit gesehen werden, Konsumgüter preiswerter als im eigenen Land einzukaufen. ${ }^{6}$ In den (strategischen) Kalkülen von Unternehmen, wie auch im eher taktischen Umgang von Konsument*innen mit den Möglichkeiten, die aus dem Aufeinandertreffen zweier staatlicher Territorien resultieren, ist die Grenze weniger eine

5 A. Yeliseyeu, Keeping the Door Ajar. Local Border Traffic Regimes on the EU's Eastern Borders, Helsinki: T. F. I. o. I. A.-U. instituutti, 2014. Die aktuellen Diskussionen um die zukünftige Kontrolle der irisch-nordirischen Grenze zeigt, wie komplex der Widerspruch von Mobilität und Kontrolle ist, wenn es um deren konkrete Gestaltung vor Ort geht.

6 Ein kleines, aber sicher nicht ganz untypisches Beispiel ist der Kauf von Feuerwerkskörpern zu Silvester durch Niederländer*innen in Deutschland. Nachdem im Jahr 2000 in der niederländischen Stadt Enschede ein Lager für Feuerwerkskörper explodiert war, wodurch ein ganzes Stadtviertel zerstört wurde, 23 Menschen starben und nahezu 1000 Menschen verletzt wurden, wurden Feuerwerkskörper durch gesetzgeberische Maßnahmen erheblich verteuert und ihr Verkauf limitiert (u. a. durch die Einführung besonderer Verkaufsstellen). Seitdem erhöht sich jährlich zu Silvester der Absatz deutscher Discounter in Grenznähe; für manche Geschäfte sind die Tage, an denen Feuerwerkskörper verkauft werden dürfen, mittlerweile die umsatzstärksten des Jahres, siehe z. B. https://rp-online.de/nrw/staedte/wesel/hollaender-kaufen-in-wesel-billigboeller_aid-13871093 (abgerufen am 24. März 2019); https://www.noz.de/archiv/vermischtes/arti kel/217965/niederlander-kaufen-im-emsland-gunstiger-ein (abgerufen am 24. März 2019); https:// www.ndr.de/nachrichten/niedersachsen/osnabrueck_emsland/Ansturm-auf-Boeller-Die-Nieder laender-kommen,silvester880.html (abgerufen am 24. März 2019). Obwohl auch die private Einfuhr von Feuerwerkskörpern verboten ist, wenn diese bestimmte Kennzeichnungspflichten nicht erfüllen, ist diese Art des Imports eine weithin akzeptierte Praxis. 
Restriktion, sondern vielmehr eine Ressource, die - je nach Perspektive - Profite oder Einsparungen ermöglicht, den Lebensunterhalt sichert oder die Befriedigung eigener Bedürfnisse ermöglicht. Gleichzeitig wird aber aufgrund dieses Ressourcencharakter ein bestimmtes Verhältnis zum jeweiligen Staat etabliert, das durch den formalen bzw. informellen Charakter der ökonomischen Tätigkeiten gekennzeichnet ist. Die Grenze zwischen regelungskonformen Praktiken und solchen, die diese Regelungen subvertieren, ist nicht immer unscharf. ${ }^{7}$

Alltägliche Aneignungen von Grenzen basieren häufig auf einer Differenz oder einem angenommenen Gefälle z. B. von Angeboten und Preisen oder von Löhnen und Gehältern. Darüber hinaus spielen aber Unterschiede in der Rechtslage eine Rolle - z. B. bezüglich der Möglichkeit, gegen Geld sexuelle Befriedigung zu erfahren. Prostitution im Grenzraum kann aber auch auf anderen Differenzen basieren: auf Machtlosigkeit oder mangelndem Interesse an der Kontrolle, auf Armut und Perspektivlosigkeit, die zur sexuellen Ausbeutung von Frauen und Kindern führen, wie auch darauf, dass Freier Grenzräume oftmals als rechtsfreie Räume betrachten. ${ }^{8}$ Mit anderen Worten: Welche Form von grenzüberschreitenden Beziehungen wie bedingt ist und welche an der Grenze manifest werdenden Differenzen jeweils konstitutive Bedeutung haben und welche Folgen dies für wen hat, kann nur im konkreten Fall bestimmt werden.

Die EU bietet zahlreiche Beispiele dafür, dass in den vergangenen Jahrzehnten Verflechtungen von Alltagspraktiken wie auch Koordinationen im administrativen und planerischen Bereich zu einem einflussreichen Narrativ der Gestaltung von Grenzländern geworden sind. Grenzüberschreitende Zusammenarbeit institutionell gefasst in Euregios/Euroregionen - gilt vielfach als Laboratorium europäischer Integration. Die Implementierung des Schengener Grenzregimes sowie die Übernahme desselben in den acquis communautaire, wodurch der „Raum der Freiheit, der Sicherheit und des Rechts“ geschaffen wurde, als den sich die EU seit dem Vertrag von Amsterdam (1999) versteht, haben diesen Prozess seit Mitte der 1980er Jahren zwar beschleunigt, seine Anfänge reichen jedoch in die 1950er Jahre zurück. Europäische Grenzländer sind somit auch Teil der Imagination und Utopie ${ }^{9}$ eines integrierten (gelegentlich auch als kosmopolitisch ent-

7 Vgl. hierzu die Beispiele zu subversiven Praktiken an Grenzen in Bruns, Miggelbrink, Subverting Borders.

8 C. Schauer, ,Jeder holt sich, was er will. Sexuelle Ausbeutung von Frauen und Kindern in einer tschechischen Grenzregion“, Osteuropa 56 (2006) 6, S. 235-244.

$9 \mathrm{Zu}$ Recht wurde darauf hingewiesen, dass Euroregionen oftmals eher symbolischer Art sind oder weitgehend elitäre Projekte, die nicht unbedingt mit den Alltagspraktiken von Menschen in Grenzregionen korrespondieren (K. Mirwaldt, „Contact, Conflict and Geography: What Factors Shape Cross-border Citizen Relations?“, Political Geography 29 [2010] 8, S. 434-443; J. M. Trillo- 
worfenen) Europas und sie sind zugleich realweltliche Labore, in denen grenzüberschreitende Praktiken entworfen, stabilisiert oder verworfen werden. ${ }^{10}$

Grenzländer sind zudem - und hier schließe ich an die Diskussion um Souveränität in Kapitel 2 an - relevante räumliche Formierungen, wenn es um die Frage geht, ob und wie unter Bedingungen der Globalisierung zunehmend postterritoriale Souveränitätsregime entstehen und dominant werden - also Souveränitätsregime, deren Funktionsweise nicht (mehr) an die Existenz der Raumformats des territorialen Nationalstaats gebunden sind, wie Agnew ${ }^{11}$ vermutet. Eine diesbezügliche Arbeitshypothese könnte lauten, dass soziale und ökonomische Verflechtungen, sofern sie sich in institutionalisierten Formen niederschlagen in der Lage sein könnten, hegemoniale Territorialisierungen zu unterlaufen und ggf. zurückzudrängen. Möglicherweise entsteht so eine neue Qualität von Territorialitäten deren Bezug zu souveränen Formen der Machtausübung zunehmend unklar werden und verschwimmen, wie Jureit und Tietze ${ }^{12}$ beobachten.

Santamaria, „Cross-Border Regions: The Gap Between the Elite’s Projects and People’s Awareness. Reflections from the Galicia-North Portugal Euroregion“, Journal of Borderlands Studies 29 [2014] 2, S. 257-273). Auch das Potenzial, demokratische Teilhabe im europäischen Kontext zu fördern, wird mittlerweile eher skeptisch beurteilt, siehe O. Kramsch, „Navigating the Spaces of Kantian Reason: Notes on Cosmopolitical Governance with the Cross-Border Euregios of the European Union“, Geopolitics 6 (2001) 2, S. 27-50; Ders., „Along the Borgesian Frontier: Excavating the Neighbourhood of ,Wider Europe““, Geopolitics 16 (2011) 1, S. $193-210$.

10 Die Erwartungen gingen durchaus über eine bloße Koordination von Planung und (lokaler) Politik hinaus; Grenzregionen schienen als der Ort, an dem die Idee einer transnationalen Staatsbürgerschaft keine abstrakte Vorstellung, sondern in der Gefühls- und Erfahrungswelt von Menschen verankert ist (vgl. E. Lissandrello, The Utopia of Cross-border Regions: Territorial Transformation and Cross-border Governance on Espace Mont-Blanc, Nijmegen, 2006).

11 Agnew, „Sovereignty Regimes“.

12 U. Jureit, N. Tietze, Postsouveräne Territorialität. Die Europäische Union und ihr Raum, Hamburg: Hamburger Edition - Verlag des Hamburger Instituts für Sozialforschung, 2015. 